See Article page XXX.

\section{Commentary: Post operative atrial fibrillation: Out of sight out of mind}

\author{
Jean-Francois Legare, MD
}

In their study, "The long-term impact of postoperative atrial fibrillation after cardiac surgery," Bianco and colleagues ${ }^{1}$ add to a growing body of evidence suggesting that postoperative atrial fibrillation (POAF) is associated with increased risk of long-term mortality. ${ }^{2,3}$ This is particularly important because POAF has been shown to affect one-third of patients who undergo cardiac surgery. In fact, some groups of patients like patients with valvular heart disease or combination of ischemic heart disease and valvular heart disease experience higher risks of POAF.

Unfortunately, the reality is that for most surgeons POAF is often thought of as part for the course for patients who undergo cardiac surgery. This is not to say that POAF is ignored but it is perceived as largely self-limited with most patients returning to normal sinus rhythm before discharge. As such this lack of an obvious effect on short-term in-hospital outcome likely contributes to a "laissez-faire" attitude. The present study, while not establishing a definitive cause and effect relationship, provides compelling evidence that POAF is associated with worse outcomes after discharge from hospital. Not surprising, this lack of cause and effect relationship has often led some authors to suggest that POAF is a marker of disease severity identifying who are at risk of long-term adverse events.

\footnotetext{
From the Department of Surgery, The New Brunswick Heart Center, Dalhousie Medicine New Brunswick, Saint John, New Brunswick, Canada.

Disclosures: The author reported no conflicts of interest.

The Journal policy requires editors and reviewers to disclose conflicts of interest and to decline handling or reviewing manuscripts for which they may have a conflict of interest. The editors and reviewers of this article have no conflicts of interest.

Received for publication Nov 16, 2021; revisions received Nov 16, 2021; accepted for publication Nov 17, 2021.

Address for reprints: Jean-Francois Legare, MD, New Brunswick Heart Center, 400 University Ave, PO Box 2100, Saint John, New Brunswick E2L 4L2, Canada (E-mail: legarej@me.com).

J Thorac Cardiovasc Surg 2021; $: 1-2$

$0022-5223 / \$ 36.00$

Copyright (c) 2021 by The American Association for Thoracic Surgery

https://doi.org/10.1016/j.jtcvs.2021.11.029
}

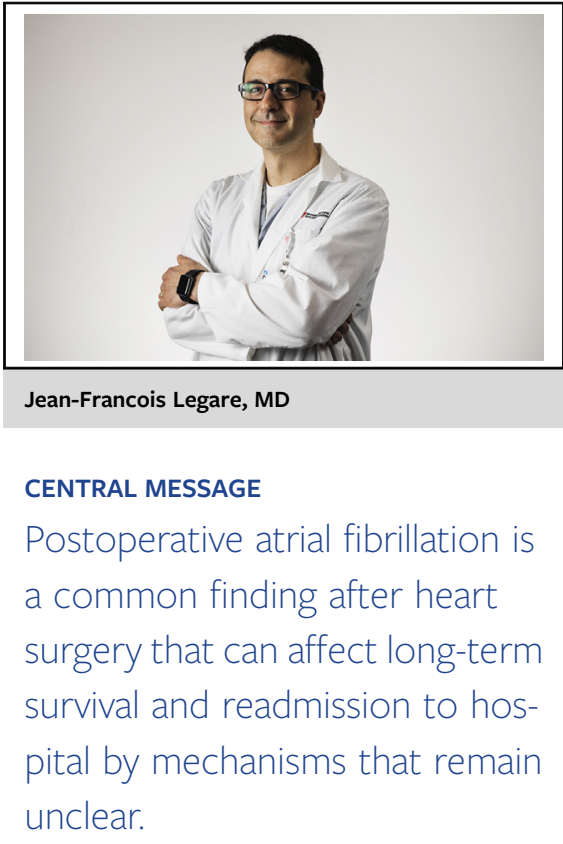

Although POAF appears to affect long-term outcomes, the mechanism responsible for this observation remains unclear. For example, there are inconsistent data regarding the effect of POAF on long-term adverse events like the risk of stroke. ${ }^{4}$ The present study despite its large size was unable to demonstrate a clear association between POAF and stroke. However, findings from the present study did suggest that POAF was associated with a significant risk for readmission to hospital because of heart failure. Perhaps this represents an important clue for a potential mechanism to explain excess mortality or adverse events in patients with POAF but would need to be explored.

What is apparent is that future breakthrough in our understanding of POAF will need to include studies that go beyond a retrospective group analysis. Our evaluation of the effect of POAF needs to shift its focus onto the major areas of knowledge gaps using prospective study designs and appropriate clinical trials. In particular the following areas come to mind:

1. Monitoring: there is currently no guidelines or standard approach to monitoring particularly with the advent of easy to use wearable devices capable of monitoring patients after discharge.

2. Predefined long-term outcome: there is currently no defined outcomes (long-term) related to POAF like 
heart failure and little standardization defining actual cause of death in patients followed after surgery.

3. Trial therapies that can affect long-term outcomes: limited efforts to date to have prospective trials capable of testing existing therapies and their effect on long-term outcomes related to POAF (heart failure therapies like angiotensin-converting enzyme, angiotensin receptor blocker, and anticoagulants).

\section{References}

1. Bianco V, Kilic A, Yousef S, Serna-Gallegos D, Aranda-Michael E, Wang Y, et al. The long-term impact of postoperative atrial fibrillation after cardiac surgery. $J$ Thorac Cardiovasc Surg. 2021. XXX:XXX.

2. Phan K, Ha HS, Phan S, Medi C, Thomas SP, Yan TD. New-onset atrial fibrillation following coronary bypass surgery predicts long-term mortality: a systematic review and meta-analysis. Eur J Cardiothorac Surg. 2015;48:817-24.

3. Thorén E, Wernroth ML. Compared with matched controls, patients with postoperative atrial fibrillation (POAF) have increased long-term AF after CABG, and POAF is further associated with increased ischemic stroke, heart failure and mortality even after adjustment for AF. Clin Res Cardiol. 2020;109:1232-42.

4. Benedetto U, Gaudino MF, Dimagli A, Gerry S, Gray A, Lees B, et al. Post-operative atrial fibrillation and long-term risk of stroke after isolated coronary artery bypass graft surgery. Circulation. 2020;142:1320-9. 